\title{
La réforme du curriculum québécois et son difficile atterrissage
}

Reform of the Quebecois curriculum and its difficult arrival La reforma del currículo quebequense y su difícil aterrizaje

\section{Claude Lessard}

\section{(c) OpenEdition}

Journals

Édition électronique

URL : https://journals.openedition.org/ries/5623

DOI : 10.4000/ries.5623

ISSN : 2261-4265

Éditeur

France Education international

Édition imprimée

Date de publication : 1 décembre 2016

Pagination : 95-106

ISBN : 978-2-85420-612-8

ISSN : $1254-4590$

\section{Référence électronique}

Claude Lessard, «La réforme du curriculum québécois et son difficile atterrissage », Revue internationale d'éducation de Sèvres [En ligne], 73 I décembre 2016, mis en ligne le 01 décembre 2018, consulté le 01 juillet 2021. URL : http://journals.openedition.org/ries/5623 ; DOI : https://doi.org/ $10.4000 /$ ries.5623

(c) Tous droits réservés 


\section{La réforme du curriculum québécois et son difficile atterrissage}

\section{Claude Lessard \\ Université de Montréal}

Le Québec a connu, au tournant du millénaire, une importante réforme du curriculum de l'éducation préscolaire et de l'enseignement primaire et secondaire ${ }^{1}$. Préparée tout au long des années quatre-vingt-dix par des avis du Conseil supérieur de l'éducation, et surtout par le travail de la commission des États généraux de l'éducation (1996), cette réforme a été implantée graduellement au début des années 2000 au primaire, puis au secondaire à compter de 2005.

Comme il est arrivé dans d'autres contextes nationaux, cette réforme a été controversée. Les débats mettant en opposition les acteurs de l'école ne furent pas différents de ceux qui déchirèrent la société civile et furent répercutés par les médias. Ils n'eurent que très peu d'échos dans le reste du Canada et aux États-Unis. Dans ces parties de l'Amérique, il y a un consensus sur une orientation pragmatique et communautaire de l'école obligatoire, qui prépare à la vie et entend insérer dans la société une personne apte à améliorer celle-ci ; aussi, le débat sur la place des humanités et sur la liaison éducation et économie n'y apparaît qu'au post-secondaire, pas avant.

$\mathrm{Au}$ Québec, une dimension politique non négligeable influa sur la réforme : conçue, élaborée et décidée sous le gouvernement d'un parti de centre gauche, elle fut mise en œuvre, notamment au secondaire, sous le gouvernement d'un autre parti, de centre droit. Au fil des ans, plusieurs ministres différents y ont été associés, compliquant de ce fait le pilotage et le monitoring ${ }^{2}$.

Les controverses entourant la réforme du curriculum - rebaptisée en cours de route "renouveau pédagogique " - sont maintenant apaisées, même si elles ne sont pas résolues. Vingt ans plus tard, un cycle de politique publique en matière de curriculum semble achevé. D'ailleurs, le Conseil supérieur de l'éducation a publié en 2014 un rapport qui se voulait non pas un bilan, mais plutôt une réflexion sur les processus de transformation du curriculum mis en

\footnotetext{
1. J'ai été tout au long de ce processus un « ami critique », i.e. que j'ai adhéré à l'entreprise et défendu ses grandes orientations exposées plus avant, même si, et comme d'autres, il a fallu reconnaître des ratés, tant dans la facture des programmes que dans les modalités de leur mise en œuvre. Dans ce texte, je propose une analyse de ce qui s'est passé, en espérant qu'on n'y percevra pas la posture du « gérant d'estrade » qui martèle " je vous l'avais bien dit ». Justement, il y a des situations où il est tout autant difficile d'être parfait du premier coup que de corriger le tir en cours de route.

2. De la commission des États généraux de l'éducation en 1995, jusqu’à aujourd'hui, le Québec a vu défiler aux affaires une quinzaine de ministres de l'éducation différents !
} 
œuvre dans le cadre de cette réforme (CSE, 2014). Aussi, le ministère de l'éducation a demandé à des chercheurs indépendants de procéder à son évaluation, tant auprès des élèves que de leurs parents (on n'a pu recueillir les perceptions des enseignants, ces derniers refusant de participer à l'étude; nous y reviendrons). Cette évaluation s'est échelonnée de 2007 à $2013^{3}$.

Une politique ou une réforme peut être découpée en deux grandes composantes : l'élaboration de son contenu, et sa mise en œuvre ou sa traduction concrète. Estimons (arbitrairement...) à moitié-moitié l'importance de ces deux éléments. En d'autres termes, une bonne politique véhicule de bonnes idées et elle est mise en ouvre avec doigté, soin et constance (Lessard, Carpentier, 2015). Il faut les deux : l'histoire de l'éducation nous apprend que de bonnes idées et des innovations intéressantes meurent faute d'avoir été soutenues convenablement. On les a "brûlées », dit-on couramment au Québec, pour indiquer qu'elles ont perdu leur potentiel de mobilisation. Aussi, la meilleure gestion du changement ne peut combler les lacunes d'idées mal conçues ou inappropriées.

Dans cet article, nous abordons ces deux composantes, rappelons les finalités formulées par la commission des États généraux et reprises par l'Énoncé de politique de 1998, puis discutons de leur mise en ouvre au cours de la dernière décennie.

\section{UNE POLITIQUE ÉDUCATIVE AMBITIEUSE}

Ce qui frappe, vingt ans après la parution du rapport de la commission des États généraux et l'Énoncé de politique de 1998, c'est l'ambition dont a fait preuve l'État québécois en matière d'éducation. Forte d'une large consultation publique qui avait permis de mobiliser la société civile autour de l'éducation, et aussi assurée d'un soutien ministériel significatif, la commission a remis un rapport présentant une vision de la mission de l'école (instruire, socialiser, qualifier), un diagnostic des déficiences et des priorités à poursuivre. Il fut repris presqu'intégralement par l'Énoncé de politique ministérielle, ce qui est une reconnaissance de sa pertinence politique. On peut parler d'une politique éducative globale, couvrant tous les ordres d'enseignement, et intégrée autour d'une double finalité d'égalité des chances et de renforcement de l'école - surtout de l'école publique - et de sa fonction cognitive et culturelle. Ces deux finalités, pas aisément conciliables, s'exprimaient dans l'objectif rassembleur d'une réussite éducative pour tous. Cet objectif fut l'objet d'un consensus social que rétrospectivement on peut qualifier de «mou».

3. Soulignons la quasi absence des universitaires dans la première étape (diagnostic, formulation des finalités, élaboration des contenus) et leur arrivée tardive dans la seconde (quasi absence dans la mise en œuvre, mais intervention dans l'évaluation des résultats). On voulait des praticiens, des acteurs du terrain, des gens d'expérience... au mépris de l'expertise universitaire. 
Une réforme suppose à la fois un regard en arrière et un regard en avant. Le premier fait le bilan du chemin parcouru, cerne les réussites et les échecs et nomme les correctifs prioritaires. Le second se tourne vers l'avenir : qu'est-il essentiel de transmettre aux jeunes et aux adultes d'aujourd'hui pour qu'ils puissent contribuer au monde tel qu'il nous est possible de le dessiner et tel que nous le désirons?

Le premier chantier de la commission formula une finalité de justice et d'équité. Il y fut proposé de "remettre l'école sur ses rails en matière d'égalité ». C'est donc dire que malgré tout le chemin parcouru en matière de démocratisation de l'école depuis la Révolution Tranquille des années soixante, des ratés et des impasses étaient constatés : des écarts de scolarisation importants entre milieux sociaux, entre garçons et filles, entre le réseau d'enseignement public et le réseau privé, voire des écarts de qualité au sein même du réseau public, l'accès en région à certains programmes - notamment en formation technique et professionnelle -, tout cela s'avérait problématique, tout comme les élèves en difficulté d'apprentissage partageant des profils socioéconomiques particuliers. Le Québec, à cause de ces réalités, se montrait incapable d'atteindre ses objectifs de diplomation au secondaire, hypothéquant ainsi l'avenir des jeunes comme celui de la collectivité.

Il fallait donc corriger le tir. Cela signifiait d'abord, intervenir dans les services à la petite enfance, généraliser la maternelle à 5 ans à temps plein. Tout en demeurant centré sur le jeu, son programme éducatif, à certaines conditions en assurant la qualité, devait être en mesure de préparer les enfants à la scolarisation. Il était particulièrement important qu'en partenariat avec les familles de milieux défavorisés, à qui la maternelle pourrait être accessible dès l'âge de 4 ans (ce qui est le cas depuis 2013), celle-ci puisse mettre ces enfants sur le même pied que les autres face à la scolarisation.

Cela signifiait ensuite soutenir les écoles primaires et secondaires œuvrant en milieu défavorisé. L'équité commandait d'investir davantage dans ces écoles, en partenariat avec la communauté environnante.

Enfin, cela impliquait de s'attaquer à la stratification croissante des écoles publiques et privées, qui, notamment dans les grandes régions urbaines, étaient en forte concurrence au secondaire, pour recruter les meilleurs élèves ${ }^{4}$. En effet, les écoles ont multiplié les "projets particuliers » et les programmes enrichis5, dans le but d'attirer et de retenir les bons élèves. Il s'en est suivi des écarts importants, aux contours socioéconomiques connus, entre écoles et au sein même des écoles, entre le secteur régulier et les parcours enrichis. La

4. Les parents québécois ont le choix entre inscrire leur enfant au réseau d'enseignement public ou au réseau privé (payant). Normalement, les parents sont censés inscrire leurs enfants à l'école publique du quartier, mais ils peuvent, s'il y a des places disponibles, les inscrire dans une école de la commission scolaire, voire d'une autre commission scolaire (s'ils acceptent d'assumer le supplément de coût lié au transport scolaire).

5. Il s'agit par exemple du programme d'éducation internationale, de programmes sports-études, d'arts-études, d'anglais intensif, etc. 
commission des États généraux proposa un moratoire sur la création d'écoles privées et l'intégration graduelle des écoles existantes au sein du réseau public. Elle recommanda aussi que les projets particuliers soient balisés et qu'ils ne puissent être sélectifs pendant la scolarité obligatoire.

L'autre grande finalité de la réforme touchait le renforcement de la fonction cognitive et culturelle de l'école. Cela passait nécessairement par une restructuration du curriculum du primaire et du secondaire, jugé trop «light» dans ses contenus (expression de l'auteur de ce texte) et peu prompt à développer chez les jeunes les compétences "générales ( (elles deviendront "transversales » en cours de route) nécessaires pour participer pleinement à un monde et à une économie qui les exigent. Restructurer le curriculum exigeait aussi d'en éliminer certaines "petites matières " (éducation au choix de la carrière, éducation sexuelle, éducation familiale) qui consommaient du temps d'enseignement, de consacrer ce temps récupéré aux matières devant être renforcées (notamment, la langue d'enseignement et l'histoire). Cela commandait aussi de réorganiser certains enseignements, comme par exemple, au premier cycle du secondaire, de regrouper les sciences et la technologie dans un seul programme intégré. Cela exigeait, enfin, d'ajouter certaines matières que le contexte actuel requiert, par exemple, un enseignement d'éthique et de culture religieuse, prenant en compte le pluralisme spirituel et religieux que l'immigration et la mondialisation accentuent.

Ce curriculum devait être commun à la très grande majorité des élèves, du moins jusqu'à la fin du premier cycle du secondaire, après quoi un jeu d'options devait être déployé en fonction des exigences d'entrée dans les institutions post-secondaires. De type tronc commun, le curriculum ne se différenciait que pour une minorité d'élèves attirés ou dirigés vers une formation professionnelle. Cette dernière était en déclin auprès des jeunes depuis plusieurs décennies et on cherchait des stratégies efficaces de revalorisation. On cherche toujours... Au second cycle du secondaire, il était prévu d'organiser un cheminement plus pratique et technologique pour des élèves censés préférer ce type d'apprentissage, mais le curriculum devait demeurer le même. Cette voie dite technologique s'est peu développée, faute de ressources, de masse critique d'élèves et d'intérêt véritable des acteurs scolaires.

En termes de finalités, la réforme posait comme mission de l'enseignement obligatoire celle d'instruire, de socialiser et de qualifier, et dans cet ordre. L'ensemble du curriculum devait être revu suivant deux grandes perspectives structurantes : une perspective de rehaussement culturel et une autre axée sur le développement de compétences générales ou transversales. La perspective «culturelle» signifiait que dans chaque discipline, les savoirs transmis devaient, d'une part, être situés dans leur contexte d'émergence, et d'autre part qu'en lien avec les domaines généraux de formation (voir plus bas), on en montre les usages dans les divers champs de l'activité humaine. Le rehaussement culturel signifiait aussi que le curriculum devait assurer à tous les élèves le partage de repères 
communs, notamment dans les humanités, puisque c'est par le partage d'une culture commune qu'une société se constitue et "tient». Le souci des compétences générales ou transversales exprimait le profil de formation recherché à la sortie de l'école : autonomie de pensée et créativité personnelle, esprit d'analyse et de synthèse, capacité de résolution de problèmes, esprit critique, méthode efficace de travail, capacité de communication et littératie technologique.

\section{LA MISE EN GUVRE}

La mise en œuvre est souvent le moment des choix difficiles et contraints. Des finalités généreuses et ambitieuses, donnant parfois la fausse impression que le consensus social est fort et durable, se heurtent à la réalité des rapports sociaux internes et externes au monde de l'éducation et aux représentations que ces acteurs ont de la bonne école et du bon curriculum. Alors, les finalités se traduisent de manière partielle ou encore, on en abandonne des éléments jugés trop onéreux ou engendrant trop de résistance. C'est ainsi qu'en ce qui a trait à la finalité de justice sociale et d'équité scolaire, l'intervention précoce fut retenue ; en son nom, on généralisa les maternelles 5 ans, on introduisit les maternelles 4 ans en milieu défavorisé, on développa un programme de soutien aux écoles de milieu défavorisé ${ }^{6}$; on maintint la gratuité scolaire à l'ordre collégial ${ }^{7}$ et des frais de scolarité universitaire sous la moyenne canadienne. En revanche, on ne toucha pas à l'enseignement privé, à son financement, à sa sélectivité, ni ne chercha à mettre un frein à sa croissance. On laissa ainsi libre cours à la concurrence entre les écoles et à la multiplication des programmes enrichis et des projets particuliers dans le réseau public. Si le Québec s'est engagé à faire plus pour les écoles et les familles en milieux défavorisés, ses classes moyennes, soucieuses de l'avenir de leur progéniture, ne semblent pas prêtes à renoncer aux avantages socio-scolaires dont elles profitent en ayant un accès privilégié au réseau privé et aux programmes enrichis du réseau public.

En ce qui concerne le curriculum, le cadre pour l'élaborer innova. Alors que les anciens programmes avaient été formulés soit en termes de contenus et de séquences de contenus à couvrir (dans les années soixante), soit en termes d'objectifs (initiaux, intermédiaires et terminaux, dans les années quatre-vingt), les nouveaux programmes furent revus et construits suivant un format reliant systématiquement trois ensembles distincts : des compétences transversales, des domaines d'apprentissage et des domaines généraux de formation. Les compétences transversales sont de différents ordres : intellectuel, méthodologique, personnel et social, et de l'ordre de la communication. Les domaines généraux de formation couvrent les champs de la santé et du bien-être, de l'orientation et de l'entrepreneuriat, de l'environnement et de la consommation, des médias,

6. Ce programme ("Agir autrement ») a été évalué par des universitaires (Janosz et al., 2010) : on a constaté que le climat de ces écoles s'est amélioré, mais que la réussite des élèves n’a pas progressé.

7. Le collégial québécois correspond aux 12 et $13^{\mathrm{e}}$ années de formation. 
et du vivre ensemble et de la citoyenneté (ce qu'ailleurs on appelle " éducation à »). En ce qui concerne les domaines d'apprentissage ou les familles disciplinaires, le programme de formation de l'école québécoise les décline toujours suivant le même format: identification de trois compétences ${ }^{8}$ (et spécification du sens de chacune, en lien avec des familles de situation variées, les composantes de la compétence, les critères d'évaluation, les attentes de fin de cycle, les processus et stratégies) ; suivent les notions et concepts à enseigner. Chaque programme disciplinaire est ainsi structuré autour de développement de compétences spécifiques et de sa contribution au développement des compétences transversales. Il y a une nette priorisation des compétences, traduisant ce que certains appelaient de leurs vœux, soit le passage d'un paradigme centré sur l'enseignement à un paradigme centré sur l'apprentissage.

Il s'est agi d'une manière nouvelle de construire les programmes, exigeant des enseignants qu'ils en comprennent l'architecture, les relations entre les parties et les concepts clés. Pour des enseignants habitués à une liste d'objectifs proches des contenus usuels, cela représenta un formidable changement de représentation du curriculum. Cela étant dit, pris éléments par éléments, le programme est accessible à un enseignant (diplômé d'université...) : par exemple, les enseignants comprennent ce que sont les domaines généraux de formation, qui existaient déjà dans l'ancien programme sous une forme ou une autre, par exemple, comme matière particulière ou comme objet d'activités spécifiques, parfois parascolaires. Ce qu'il y avait de nouveau, c'est qu'ils devaient s'en soucier dans leur propre enseignement disciplinaire. Les enseignants ont aussi compris le sens des compétences transversales, en tant que finalités ou profils de sortie de la scolarisation obligatoire; c'est leur évaluation qui ne leur apparut pas évidente. Ils ont aussi saisi la réorganisation des savoirs dans certains champs, comme en science au premier cycle du secondaire. Mais, comprendre des éléments et les assimiler à du déjà connu n'est ni saisir le virage ambitieux souhaité, surtout si les concepts clés ne sont pas «stabilisés » (Qu'est-ce qu'une compétence ? Qu'estce que rehausser culturellement son enseignement?), ni y adhérer sans réserve ni savoir travailler à leur traduction concrète. Il est ainsi rapidement apparu que cette vaste et abstraite construction curriculaire n'était pas évidente à appréhender et à traduire en processus de planification de son propre enseignement. Cela se cristallisa autour des enjeux d'évaluation des compétences et autour d'un débat surréaliste sur la place respective des compétences et des connaissances.

Pour faciliter la mise en œuvre d'une « approche par compétences », le ministère refusa, dans un premier temps, de produire du matériel didactique spécifique. Il voulait couper le cordon de sécurité traditionnelle des enseignants qui leur permettait de s'en remettre au manuel, au lieu de maîtriser le programme et de développer leurs propres activités. Il en appela au professionnalisme des

8. Bien évidemment, le contenu des trois compétences varie suivant les disciplines, mais il n’y en a jamais moins ou plus de trois. On se demande pourquoi. 
enseignants et les enjoignit de construire, de préférence en équipe de cycle, leur matériel, en même temps qu'ils devaient exercer pleinement leur jugement professionnel, notamment dans l'évaluation des compétences. Il ne fallut pas trop de temps pour que l'industrie du matériel didactique ne trouve parmi les enseignants désemparés des alliés dans leur reconquête de ce marché. Et du matériel fut produit, mais avec pas mal de retard.

Le curriculum comprenait aussi une autre innovation : organisés en cycles pluriannuels, les apprentissages devaient se répartir dans un temps plus long que celui du degré annuel, leur évaluation devait être progressive et formative, le redoublement aboli ou exceptionnel, et le travail des enseignants devait devenir plus collégial. Le professionnalisme des enseignants devait être collectif. Cette idée exigeait, au plan du travail enseignant, une modification de sa représentation traditionnellement individualiste et centrée sur la classe conçue comme un univers privé, ainsi qu'une réorganisation du temps, afin d'aménager des moments de concertation. Au primaire, cette idée a fait un peu de chemin, mais le secondaire y demeure structurellement plus imperméable.

Les vifs débats, fortement médiatisés, entraînèrent le constat que ce nouveau curriculum souffrait d'un déficit de légitimité, tant au plan cognitif, moral que pragmatique (Suchman, 1995). La réponse du ministère à cette difficulté d'atterrissage prit plusieurs formes, à la fois sur le programme lui-même et aussi sur le processus de mise en œuvre.

En ce qui a trait au programme, on a réécrit celui de l'école primaire ; on a aussi élaboré une politique d'évaluation des apprentissages, mais avec un bon décalage temporel entre les deux. Plus tard, en réponse au débat sur l'importance relative des compétences et des connaissances, le ministère fit la liste des savoirs à enseigner et les classa dans de grands tableaux suivant que leur transmission était obligatoire ou facultative (on nomma cette opération « la progression des apprentissages »). Cette classification des savoirs essentiels à transmettre a été élaborée par la direction des programmes du ministère, en fonction des disciplines et des degrés et cycles d'enseignement; elle marque, pour les parents inquiets, le retour des connaissances et pour les enseignants, le retour à des directives précises en matière de contenus désormais annualisés à l'intérieur des cycles, comme si l'on remettait à la mode du jour d'anciennes façons de faire détaillant avec beaucoup de soin les contenus à enseigner. Aussi, suite à un débat sur le bulletin (d'abord centré sur les compétences et l'appréciation qualitative (par lettre) de leur degré de développement), on réintroduisit des pourcentages. Enfin, afin d'aider les enseignants ne sachant comment travailler des compétences, on conçut des "situations d'apprentissage et d'évaluation » (SAE), i.e. des situations didactiques encadrant des tâches devant être effectuées par l'élève et lui permettant de développer une ou des compétences disciplinaires et transversales, et permettant à l'enseignant d'en assurer le suivi et l'évaluation. Selon les experts, une bonne SAE comprend les étapes suivantes : l'activation des connaissances antérieures, la recherche, l'explicitation, l'institutionnalisation, la 
familiarisation et le réinvestissement, et enfin, la complexification (l'application). On a ainsi proposé aux enseignants une nouvelle façon d'enseigner, dite "socioconstructiviste », et que plusieurs ont compris comme une pédagogie de la découverte. Les conseillers pédagogiques furent appelés à travailler au développement de ces situations et à développer chez les enseignants les compétences afférentes. On peut trouver sur internet des sites rendant accessibles des banques de SAE (par exemple : evaleta.scedu.umontreal.ca) ${ }^{9}$.

$\mathrm{Si}$, au départ, le nouveau curriculum apparaissait à plusieurs difficile à travailler, assimilable à une recette de cuisine qui listerait les ingrédients, et rien d'autre (pas d'étapes à suivre, ni d'indications sur leur mélange et leur cuisson, etc.), en cours de route, on tenta de remédier aux manques, non sans regret (on aurait souhaité les enseignants plus autonomes et plus professionnels). Ce faisant, on donna prise à la perception que la réforme n'était pas bien assurée dans ses fondements, qu'elle avait été "improvisée » et qu'on était obligée de corriger ce qui avait été mal conçu au départ. Aussi et peut-être plus important, par la formulation détaillée de la progression des apprentissages souhaitée et par les SAE, on donna prise à l'accusation de «formater " l'enseignement, d'imposer une et une seule façon d'enseigner et d'évaluer et d'ainsi (paradoxalement...) réduire l'autonomie professionnelle des enseignants que l'on désirait justement accroître. Cette réaction demeure encore à ce jour très présente parmi les responsables syndicaux du secondaire.

La mise en œuvre du curriculum, puis ses corrections, se firent à l'échelle du système entier ; il n'y eut pas d'expérimentations ou d'expériences pilotes à échelle réduite, puis évaluées et généralisées. Pourtant, le CSE (1998) avait recommandé que l'introduction des compétences transversales fasse l'objet d'une expérimentation et qu'elle soit « un point de départ de travaux multiformes de développement du curriculum plutôt qu'une consigne définie une fois pour toutes ». Comme souvent, des impératifs politiques ont ici primé : il fallait aller de l'avant, engranger les bénéfices politiques pendant qu'il était temps, rendre irréversible le nouvel ordre des choses, quel que soit le gouvernement en place. Le Conseil estimait que "l'une des façons les plus menaçantes de "brûler l'idée" serait de ne pas en reconnaître la difficulté et de négliger d'y travailler patiemment avec les enseignants et les enseignantes des disciplines les plus diverses » (1998). Difficile de ne pas lui donner raison.

Au plan de la gouvernance du changement, on mit sur pied un comité conseil, une table nationale de pilotage regroupant les principaux acteurs, et un comité conseil des programmes devant «valider» les programmes d'études tels qu'élaborés par les équipes ministérielles. Sur papier, il y avait là des dispositifs

9. On ne sait pas de manière systématique jusqu'à quel point les enseignants utilisent ces SAE, quelle place elles occupent dans leur enseignement et ce qu'ils en pensent. S'agit-il pour eux d'une nouvelle forme de travaux pratiques, enrobés de cognitivisme et de socioconstructivisme ? Sont-ce des accessoires parmi d'autres ou le cœur de leur enseignement? Comment cela se passe-t-il dans les diverses disciplines? Dans son avis de 2014, le CSE recommandait la mise sur pied d'un observatoire du curriculum, pour être en mesure de saisir le curriculum enseigné. 
pertinents. Mais ils connurent des ratés : la table nationale, de l'avis de plusieurs, devint rapidement un lieu de négociation patronale-syndicale, dysfonctionnelle et incapable de dépasser la prise en compte des irritants les plus médiatisés; le comité conseil des programme apparut assez tôt comme une nouveauté institutionnelle qui avait besoin de temps pour apprendre son rôle ${ }^{10}$, et le temps pressait... À proprement parler, les nouveaux programmes disciplinaires n'ont pas été sérieusement validés auprès des enseignants et de leurs associations.

On développa aussi une stratégie de communication qui engageait des centaines de directions et de conseillers pédagogiques à parfaire leur formation lors de grandes réunions annuelles, puis à diffuser la «bonne parole » dans leurs milieux respectifs. Mais il a manqué des ressources d'accompagnement et de proximité, un manque qui n'était pas de nature à soutenir, voire accroître, le sentiment de compétence des enseignants.

Ces ratés, dans le curriculum lui-même comme dans les processus de mise en œuvre, devinrent plus problématiques au fur et à mesure que l'on gravissait les degrés du système. Lorsque le nouveau curriculum arriva au secondaire, le ministère décida de parler dorénavant non pas de réforme du curriculum, mais de "renouveau pédagogique ». Ce qui apparaît étonnant, quand on connaît la culture du secondaire et l'importance qu'on y accorde aux contenus. Voulait-on indiquer que, dorénavant, ce n'était plus le curriculum que l'on désirait rénover, mais la "pédagogie»? Qu'il y a un lien entre ces deux dimensions, à expliciter et à renforcer ? Le sens de cette modification du vocabulaire est demeuré obscur et cela n'a pas aidé à dissiper les malentendus et la perception que le ministère entendait réduire l'autonomie professionnelle des enseignants du secondaire et formater l'enseignement. Il a aussi donné à penser que le rehaussement culturel des programmes n'était plus prioritaire, ce qui heurta bon nombre d'enseignants qui considéraient cette dimension comme une source primordiale de leur valorisation professionnelle.

Tout cela rendit l'évaluation du nouveau curriculum très problématique. Lorsque le ministère s'y mit et en confia à des universitaires le mandat, cette évaluation fut boycottée par les syndicats d'enseignants, de telle sorte que nous ne disposons pas d'une mesure scientifique des perceptions des enseignants. L'évaluation a permis aux directions et aux parents de faire connaître leurs perceptions ; dans le cas des élèves, différenciés suivant qu'ils appartenaient à une cohorte d'avant ou d'après la mise en œuvre du curriculum, leurs apprentissages ont été mesurés. Et les résultats ne sont pas sans soulever des inquiétudes et des questions ${ }^{11}$.

\footnotetext{
10. L'expertise curriculaire est rare et celle en didactique est, par définition, spécialisée.

11. Ne sachant ce que les enseignants de ces élèves ont compris, accepté et incorporé du nouveau curriculum dans leur enseignement, il est difficile d'interpréter ces résultats. Il est discutable d'opposer deux blocs homogènes, celui des enseignants hors réforme, parce que celle-ci n'était pas encore en place, et celui des enseignants dans la réforme, avec comme hypothèse qu'au sein de ce second bloc, on enseignait de manière intégrale et uniforme le nouveau programme. Il y a des enseignants d'avant la réforme qui étaient proches de son esprit, et il y a des enseignants après la réforme qui n'y ont pas adhéré.
} 


\section{Des politiques QUI S'AJOUTENT ET BROUILLENT LA MISE EN GUVRE DU CURRICULUM}

L'élaboration et la mise en ouvre du nouveau curriculum durent composer avec d'autres politiques publiques dont l'impact fut considérable dans les écoles. Deux retiennent particulièrement l'attention : d'abord, la politique d'intégration en classe régulière des élèves handicapés et en difficulté d'adaptation et d'apprentissage (EHDAA). Ce mouvement qui débuta dans les années 1970-1980, connut, au tournant du millénaire, une accélération, impulsée par le renouvellement de la politique. Il s'agit d'une tendance lourde qui a transformé la composition de la classe dite régulière et a perturbé le travail des enseignants ainsi que leur conception du métier ( «suis-je un enseignant qui transmet des connaissances et développe des compétences (surtout cognitives) ou un éducateur qui socialise des enfants dont le développement sur divers plans (autres que scolaires) est problématique ?»). Au moment où les écoles privées écrèment les écoles publiques de leurs bons éléments ${ }^{12}$ et où les commissions scolaires rivalisent de programmes enrichis et de projets particuliers pour retenir ces bons élèves, la classe régulière s'est trouvée tirée vers le bas, en même temps que sa composition se diversifiait, avec un nombre croissant d'élèves en difficulté, autrefois relégués à des classes ou des écoles dites "spéciales ». L’intégration des EHDAA a donc concurrencé la réforme du curriculum pour l'attention des enseignants et à plusieurs endroits, elle est devenue l'enjeu majeur, la source de difficultés accrues dans l'accomplissement de la tâche et dans les relations avec les parents. Le volume de l'intégration et la diversité des cas ont été tels que l'injonction faite aux enseignants de différencier leur pédagogie apparut déconnectée des conditions réelles du travail enseignant.

L'autre élément de politique publique venu brouiller l'horizon et ajouter une couche de préoccupations fut l'institutionnalisation de la gestion axée sur les résultats. L'administration publique prit ce virage au tournant du millénaire et les institutions scolaires durent suivre, notamment à compter de 2008, lorsqu'une ministre imposa des cibles de rendement (dans cinq domaines ou dimensions), incorporées dans des conventions de partenariat entre le ministère et les commissions scolaires, et aussi dans des conventions de gestion entre ces mêmes commissions scolaires et leurs écoles. Cette planification stratégique alignant les trois paliers du système éducatif commanda un important travail aux cadres scolaires. Là aussi, on peut se demander si les ambitions du nouveau curriculum ne furent pas ainsi oubliées ou réduites à une simple amélioration quantitative de la réussite scolaire, sans que celle-ci ne soit véritablement

12. Le secteur privé reçoit peu d'élèves en difficulté, notamment des élèves dont le comportement est problématique. S’il en reçoit et que cela se passe mal, il lui est toujours loisible de les retourner au public. 
rehaussée. Puisque la gestion axée sur les résultats valorise la transparence, les réalisations des commissions scolaires et des établissements sont publiques et donnent lieu à des classements. Ainsi, ce type de gestion accentue la concurrence. Certains dénoncent même une pression forte pour faire " passer » des élèves, alors que normalement ils devraient échouer.

Cet empilement de politiques et de changements multiplient les demandes auprès des directions et des enseignants et rivalisent pour leur attention et leur action.

\section{UNE « FATIGUE » QUI A DES AIRS DE RENONCEMENT POLITIQUE}

La plupart des personnes en autorité ne souhaitent pas " rouvrir »le dossier du curriculum. Les hommes et les femmes politiques actuels rivalisent de promesses à l'effet que s'ils (ou elles) sont aux affaires, ils n'imposeront pas au système éducatif une nouvelle réforme d'envergure. Ces promesses s'étendent aussi parfois aux enjeux de gouvernance et aux rapports entre les paliers et les acteurs du système. Mais si on refuse de toucher au curriculum et à la gouvernance, il reste peu de matière à une véritable politique éducative d'envergure nationale : on laisse dès lors la voie libre aux seuls enjeux de répartition des ressources et au laisser faire des forces sociales, qui tentent toujours de tirer leur épingle du jeu dans un contexte de plus en plus marchand.

Certains acteurs politiques estiment - sans le dire - que le ministère a laisser échapper le ballon, qu'il aurait raté une belle occasion, une entreprise au départ bien reçue par la population. Il ne faut donc pas compter sur lui pour faire avancer les choses. D'autres estiment que le monde de l'éducation est paralysé par des groupes d'intérêt trop forts. Ces jugements globaux ne sont pas sans fondement mais il importe de constater qu'à terme, c'est la capacité de l'État québécois de se doter d'une politique éducative qui est en cause. Une capacité proprement politique, au sens le plus noble du terme. On ne peut donc que souhaiter que cette "fatigue» soit passagère ${ }^{13}$. D'autant plus qu'avec le temps et du travail, certaines idées apparaissent sous un jour plus acceptables aux opposants, moins menaçantes aux résistants et plus fécondes aux craintifs. Aussi, ceux qui les font avancer développent avec le temps davantage d'expertise, apprennent de l'épreuve du terrain et composent avec ses contraintes. Ces idées peuvent alors entrer dans une période d'institutionnalisation plus profonde et plus réflexive.

13. Dans pareil contexte, il ne faut pas se surprendre si l'avis du CSE (2014) sur le curriculum est à toutes fins pratiques, passé inaperçu, ne donnant lieu à aucune réaction ministérielle ou politique. Pourtant, le conseil exprimait bien l'air du temps : il souhaitait non pas une reprise d'une réforme globale et ambitieuse mais plutôt des petits pas, patiemment faits avec les enseignants des diverses disciplines, pour une amélioration continue des programmes (tel est le titre de l'avis). 
Mais peut-être ces propos sont-ils trop pessimistes. Le ministre actuel a annoncé son intention de travailler, au cours de cette année scolaire, à l'élaboration d'une nouvelle politique éducative. Il n'a pas l'intention de discuter de réaménagements structurels mais tout le reste semble l'intéresser, pourvu que cela contribue à la réussite du plus grand nombre. À suivre, donc...

\section{BIBLIOGRAPHIE}

Commission des États Généraux de l'éducation (1996) : Rapport final : Rénover notre système d'éducation. Dix chantiers prioritaires, Québec : ministère de l'Éducation.

CSE (2014) : Pour l'amélioration continue du curriculum et des programmes d'études. Rapport sur l'état et les besoins de l'éducation 2012-2014, Québec: Conseil supérieur de l'éducation.

CSE (1998) : Pour un renouvellement prometteur des programmes à l'école, Québec: CSE.

CORBO C. (1994) : Préparer les jeunes au XXI siècle. Rapport du groupe de travail sur les profils de formation au primaire et au secondaire, Québec: Ministère de l'éducation.

INCHAUSPÉ P. (1997): Réaffirmer l'école. Rapport du groupe de travail sur la réforme du curriculum, Québec : ministère de l'Éducation.

JANOSZ M., BÉLANGER J., DAGENAIS C., et al. (2010) : Aller plus loin, ensemble: synthèse du rapport final d'évaluation de la stratégie d'intervention Agir autrement, Montréal, QC: Groupe de recherche sur les environnements scolaires, Université de Montréal.

LAROSE S., DUCHESNE S. (2014) : Perceptions de l'enseignement et réussite éducative au secondaire: une analyse comparative selon que les élèves ont été exposés ou non au renouveau pédagogique. Projet ERES, Université Laval. Rapport final déposé à la direction de la recherche et de l'évaluation, Québec: Ministère de l'éducation, du loisir et du sport.

LESSARD C., CARPENTIER A. (2015) : Les politiques éducatives. La mise en ouvre, Paris : PUF (Éducation et sociétés).

MAROIS P. (1997) : Prendre le virage du succès. Plan d'action ministériel pour la réforme de l'Éducation, Québec: Ministère de l'éducation, février 1997.

MEQ (2001) : Programme de formation de l'école québécoise. Éducation préscolaire et enseignement primaire. Version 2001. Québec : Ministère de l'éducation.

MEQ (2003) : Programme de formation de l'école québécoise. Enseignement secondaire, premier cycle, Québec: Ministère de l'éducation.

SUCHMAN M. (1995): "Managing Legitimacy: Strategic and Institutional Approaches ", Academy of Management Review, vol. 20, p. 571-610. 